\title{
Diagnóstico diferencial y cirugía mínimamente invasiva de un tumor suprarrenal de hallazgo antenatal
}

\author{
Differential diagnosis and minimally invasive surgery of an antenatal adrenal mass
}

\author{
Montserrat Lemus Marcenaroa, José Manuel Campos Varasa, José Fernando Vuletin Solís \\ Sergio González Bombardiere ${ }^{\mathrm{b}}$, Juan Carlos Pattillo Silva ${ }^{\mathrm{a}}$
}

\begin{abstract}
aSección Cirugía Pediátrica, División de Cirugía, Escuela de Medicina, Facultad de Medicina, Pontificia Universidad Católica de Chile bDepartamento de Anatomía Patológica, División de Imágenes, Laboratorios y Patologías, Escuela de Medicina, Facultad de Medicina, Pontificia Universidad Católica de Chile
\end{abstract}

Recibido: 17 de julio de 2018; aceptado: 28 de febrero de 2019

\begin{abstract}
Resumen
Introducción: Las masas suprarrenales en recién nacidos son infrecuentes. El diagnóstico diferencial incluye masas benignas (hemorragia suprarrenal o secuestro pulmonar extralobar) y malignas (neuroblastoma), y pueden ser un hallazgo durante la ecografía obstétrica. El uso de imágenes complementarias en el periodo postnatal permite una mejor aproximación diagnóstica, con implicancias en el manejo de estos pacientes. Objetivos: comunicar el caso de una recién nacida portadora de una masa suprarrenal, discutir los diagnósticos diferenciales y el manejo de lesiones suprarrenales en recién nacidos. Caso Clínico: Lactante de 2 meses de edad, derivada para estudio de tumor suprarrenal de diagnóstico antenatal a las 22 semanas de edad gestacional. El estudio imagenológico con ecografía postnatal mostró un tumor compatible con neuroblastoma. Paciente asintomática, estudios de laboratorios sin hallazgos relevantes. Se realizó resección laparoscópica de la lesión. El estudio histológico confirmó un secuestro pulmonar. Conclusión: El secuestro pulmonar extralobar debe ser considerado en el diagnóstico diferencial de una masa suprarenal del recién nacido. La cirugía mínimamente invasiva debiera considerarse como el abordaje de elección en casos como este, donde existe factibilidad técnica y beneficios en la recuperación y secuelas cosméticas del paciente.
\end{abstract}

\begin{abstract}
Introduction: Adrenal masses are uncommon in newborns. The differential diagnosis includes benign masses (adrenal hemorrhage, extralobar pulmonary sequestration) and malignant ones (neuroblastoma) that may be a finding during an obstetric ultrasound. The use of complementary imaging methods allows a better diagnosis approach during the postnatal period, with implications for the
\end{abstract}

Palabras clave: Neuroblastoma; tumor suprarrenal; secuestro pulmonar extralobar; niños

\section{Keywords:} neuroblastoma; adrenal tumor; extralobar pulmonary sequestration; children 
management of these patients. Objective: To report the case of a female newborn with diagnosis of an adrenal mass, and to discuss differential diagnoses and management alternatives of adrenal lesions in newborns. Case report: Two-month-old female infant, referred for adrenal tumor study diagnosed at 22 weeks gestational age. Postnatal ultrasound showed a tumor compatible with neuroblastoma. The patient was asymptomatic, and the laboratory studies showed no relevant findings. The lesion was excised by laparoscopy. A histological study confirmed pulmonary sequestration. Conclusions: Extralobar pulmonary sequestration should be considered in the differential diagnosis of an adrenal mass in the newborn. Minimally invasive surgery should be the preferred surgical technique choice in these cases, given the technical feasibility and benefits in the recovery and cosmetic issues of the patient.

\section{Introducción}

El diagnóstico prenatal de tumores suprarrenales es cada vez más frecuente debido a la amplia disponibilidad y al uso protocolizado de ecografía obstétrica ${ }^{1,2}$. Las masas suprarrenales tienen una incidencia de 1,9:1.000 recién nacidos ${ }^{3}$. El diagnóstico diferencial más importante es el neuroblastoma (NB), neoplasia maligna que, en $90 \%$ de los casos es de origen suprarrenal ${ }^{3,4}$. Otros diagnósticos diferenciales se detallan en la tabla $1^{3-5}$. El estudio de elección durante el periodo postnatal es la ecografía abdominal, la que puede complementarse con tomografía computada (TC) o resonancia magnética (RM) en caso de sospecharse malignidad ${ }^{3-5}$. Así, es posible discriminar el origen de la lesión en un $81-85 \%$ de los casos ${ }^{3}$. El manejo conservador, con seguimiento ecográfico y el uso de marcadores séricos, constituye una aproximación terapéutica razonable, aunque esto sigue siendo materia de debate ${ }^{6-9}$.

El objetivo de este artículo es comunicar el caso de una recién nacida portadora de una masa suprarrenal de diagnóstico antenatal, que fue tratada exitosamente con cirugía mínimamente invasiva. El estudio histopatológico informó un secuestro pulmonar extra lobar (SPE). Se discuten los diagnósticos diferenciales y las alternativas de manejo posibles.

\section{Caso clínico}

Lactante de 2 meses, portadora de una masa suprarrenal detectada en ecografía obstétrica a las 22 semanas de embarazo. Se identificó una masa suprarrenal izquierda, hipoecogénica, de 22 x $16 \mathrm{~mm}$ (figura 1a). Madre sin antecedentes mórbidos, multípara de 2, embarazo fisiológico bien controlado. En seguimiento ecográfico a las 35 semanas de edad gestacional se confirmó la presencia de una masa infradiafragmática, hipoecogénica, de aproximadamente $20 \times 20 \mathrm{~mm}$ aparentemente separada de la glándula suprarrenal izquierda (figura 1b). Parto vaginal a las 38 semanas, peso adecuado para la edad gestacional, sin morbilidad perinatal. Examen físico normal, sin masas abdomina- les palpables, genitales femeninos sin signos de virilización ni hiperpigmentación.

A las 7 semanas se controlaron catecolaminas urinarias, que estaban en rango normal alto para la edad (Metanefrina $206 \mu \mathrm{g} / \mathrm{g}$ creatinina y Normetanefrina $1.250 \mu \mathrm{g} / \mathrm{g}$ creatinina), decidiéndose derivación para estudio y manejo.

Se completó el estudio funcional del tumor con niveles plasmáticos de metanefrinas, testosterona, aldosterona, renina, cortisol, androstenediona, adrenalina, noradrenalina y dopamina, todos dentro de límites normales.

El estudio preoperatorio se completó con ecografía abdominal que mostró una lesión sólida, homogénea, ecogénica respecto a tejido adyacente, de bordes parcialmente definidos, sin calcificaciones evidente, que medía aproximadamente 2,7 x 1,2 x 1,7 cm. Sin flujo vascular anormal al Doppler. En el aspecto anterior de esta masa, se identificaba parcialmente un segmento de glándula suprarrenal (figura 2). Se complementó el estudio con RM que mostró una masa solida de 21 $\mathrm{mm}$ en la región suprarrenal izquierda (figura 3). No presentaba alteraciones vasculares.

Se realizó una resección del tumor por vía laparoscópica sin incidentes. Bajo anestesia general y con la paciente en decúbito lateral izquierdo $45^{\circ}$, se realizó una exploración laparoscópica con trócar umbilical de $5 \mathrm{~mm}$ insertado con técnica abierta, un trocar auxiliar de $5 \mathrm{~mm}$ en FID, y dos instrumentos de trabajo de $3 \mathrm{~mm}$ insertados de forma percutánea. Se decoló el colon, liberando el ángulo esplénico y accediendo a la transcavidad. Se identificó una masa suprarrenal adyacente a la glándula, bien delimitada, de consistencia gomosa, con irrigación discretamente aumentada. Se realizó disección y resección completa de la lesión que se extrajo a través del ombligo (figura 4). La paciente fue monitorizada por $24 \mathrm{~h}$, reinició lactancia materna en el postoperatorio inmediato, y fue dada de alta en buenas condiciones a las $48 \mathrm{~h}$. Durante seguimiento alejado a las 2 y 6 semanas se encontraba en buenas condiciones, sin evidencia ecográfica de recidiva tumoral ni otras complicaciones locales. 
Tabla 1. Diagnóstico diferencial de masas suprarrenales

\begin{tabular}{lll}
\hline & Benignas & Malignas \\
\hline Fetales & Hiperplasia suprarrenal congénita & Neuroblastoma \\
& Secuestro pulmonar extralobar & Nefroma mesoblástico congénito \\
& Duplicación renal con displasia parcial de polo superior renal & Tumor de Wilms \\
& Obstrucción renal - urinoma & \\
& Quistes duplicación gástrica & \\
& Quistes esplénicos & Neuroblastoma \\
& Adenoma suprarrenal & Ganglioneuroblastoma \\
& Ganglioneuroma & Paraganglioma \\
& Hiperplasia suprarrenal congénita & Feocromocitoma \\
& Secuestro pulmonar extralobar & Carcinoma adrenal \\
& Hemorragia/hematoma suprarrenal & Teratoma \\
& Duplicación intestinal & \\
& Quiste esplénico & \\
& Duplicación renal con displasia parcial & \\
& Urinoma \\
& Mielolipoma \\
& Quistes suprarrenales
\end{tabular}

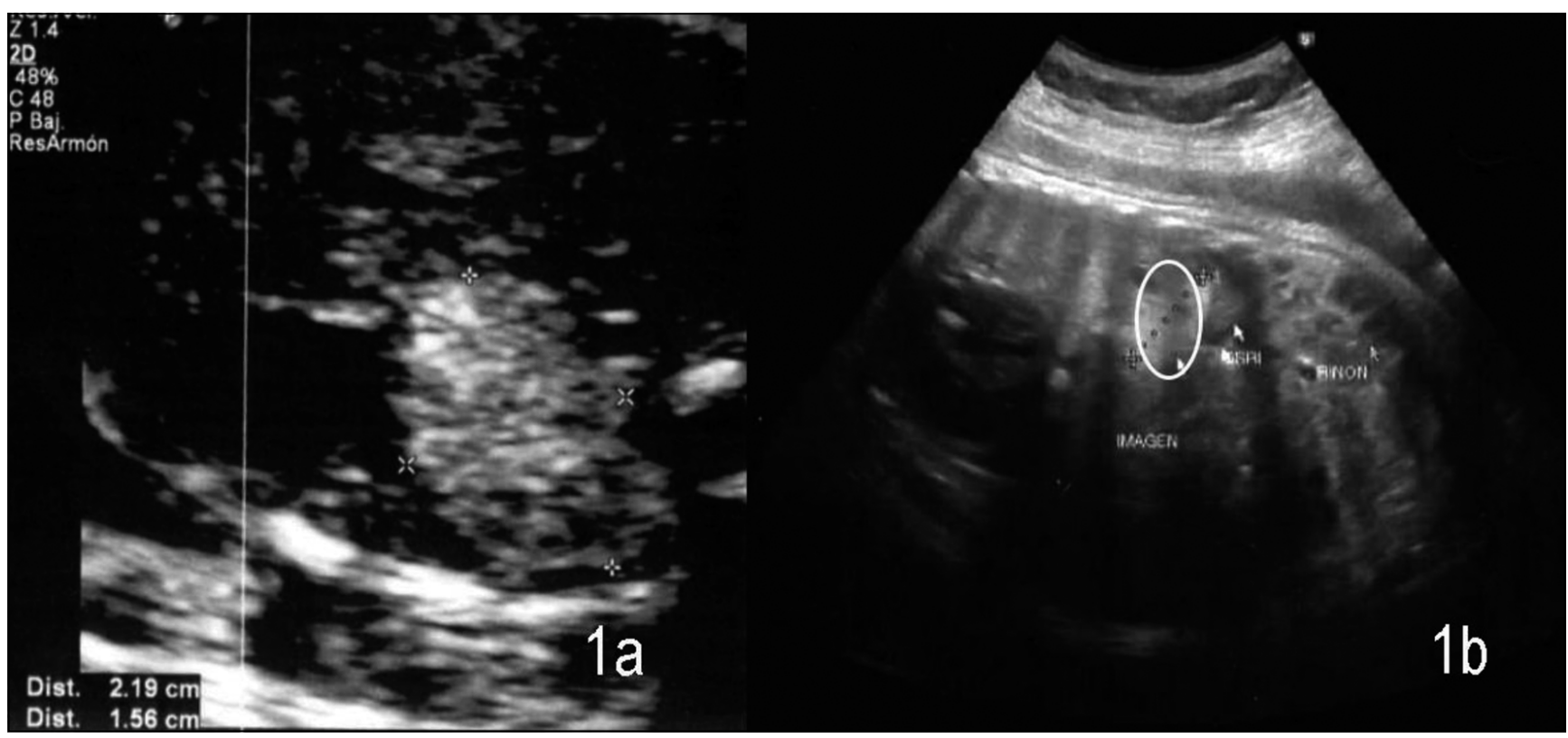

Figura 1. Ecografía prenatal donde se identifica la lesión en diferentes edades gestacional. 1a. Ecografía a las 23 semanas de edad gestacional, muestra masa hipoecogénica de 22×16 mm. 1b. Ecografía a las 35 semanas, se identifica masa hipoecogénica infradiafragmática en relación al riñón de 20 × $20 \mathrm{~mm}$ (elipse blanca).

El estudio histopatológico mostró una lesión constituida por tejido pulmonar con arquitectura de tipo fase canalicular, en la que se reconocen estructuras bronquiolares inmaduras con epitelio respiratorio, algunos conductos alveolares con macrófagos; no se identificaron alvéolos. Estroma con fibroblastos y haces de músculo liso. Algunos espacios mayores con epitelio respiratorio y cartílago hialino inmaduro. No se observó evidencia de malignidad. Estos hallazgos son compatibles con secuestro pulmonar (figura 5). 


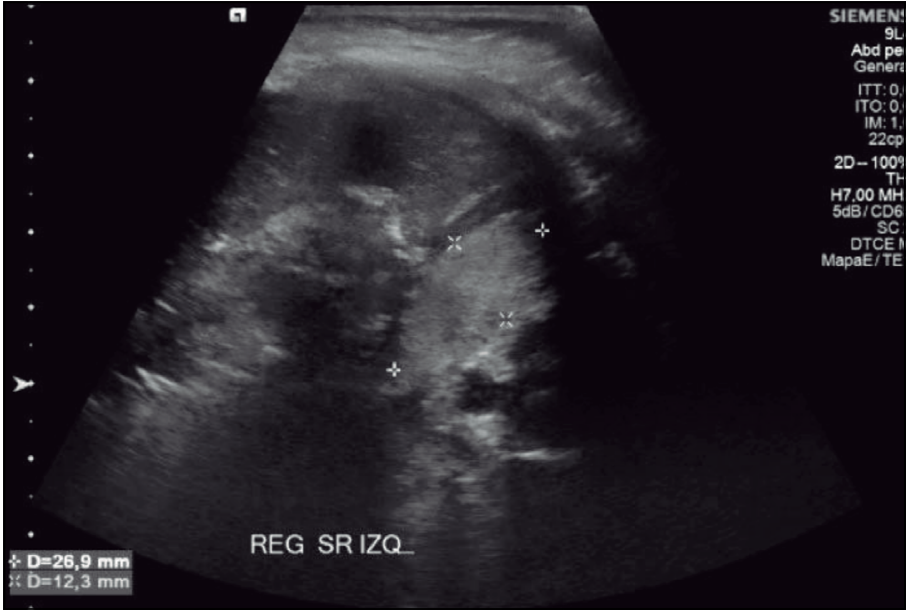

Figura 2. Ecografía abdominal a las 7 semanas de vida. Se confirma el hallazgo de masa infradiafragmática, ecogénica, sin flujo al doppler de bordes bien definidos.

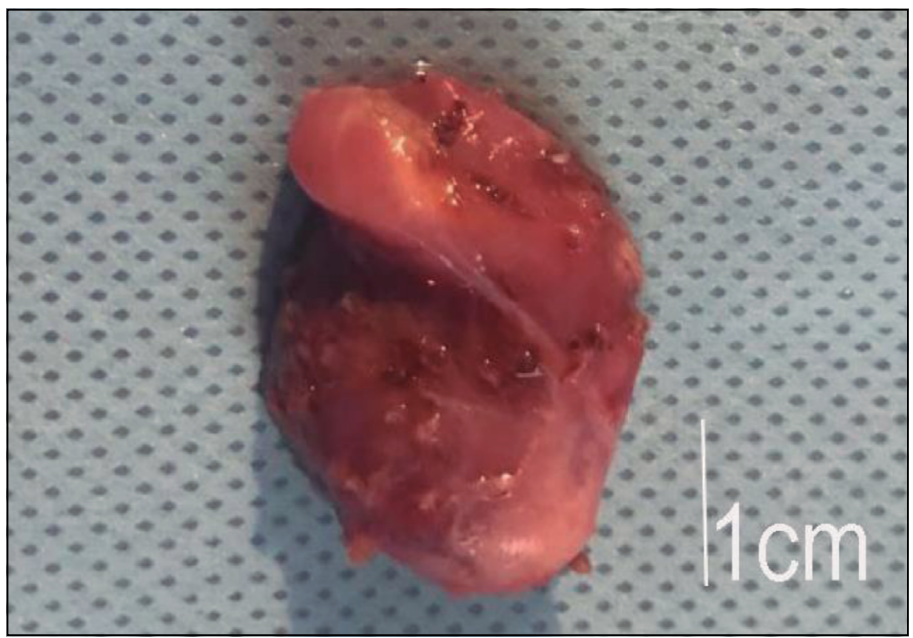

Figura 4. Pieza operatoria, es posible observar una masa bien definida, lobulada, que fue resecada por completo.

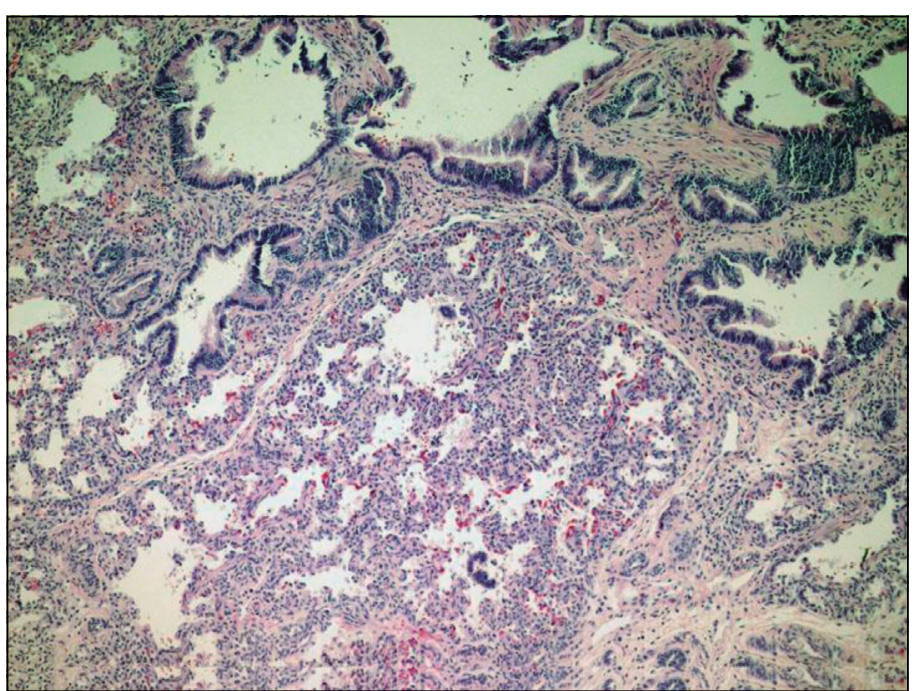

Figura 5. Microfotografía que muestra áreas de diferenciación alveolar y bronquiolar con engrosamiento de los septos alveolares y fibrosis alrededor de la vía aérea anormal. HE, 80.

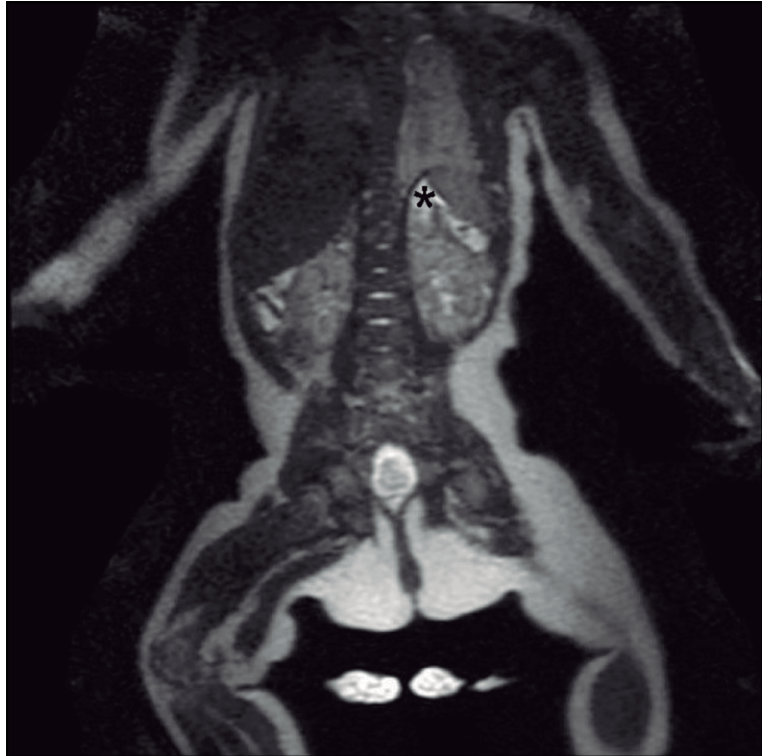

Figura 3. Resonancia magnética preoperatoria de la lesión suprarrenal. La lesión suprarrenal marcada con $\left(^{*}\right)$.

\section{Discusión}

La incidencia de masas suprarrenales sigue una distribución bimodal, siendo más frecuentes en niños menores de 5 años, y luego a partir de la cuarta y quinta década de vida ${ }^{10}$. En recién nacidos es importante establecer el diagnóstico diferencial entre NB, hemorragia suprarrenal $(0,2 \%$ de recién nacidos) y otras lesiones como el SPE, que son aun más infrecuentes ${ }^{4}$. El feocromocitoma y ganglioneuroma son tumores de niños en edad escolar, mientras que el adenoma suprarrenal, si bien es raro en niños, se presenta antes de los 5 años o después de los $10^{11}$.

La mayoría de los tumores suprarrenales se presentan como un hallazgo prenatal o durante la infancia. También pueden manifestarse como masa palpable, dolor abdominal, hipertensión arterial secundaria, falla renal, pubertad precoz, fiebre o shock, dependiendo de su origen ${ }^{12}$.

El estudio de imagen de elección para evaluar masas suprarrenales en edad pediátrica es la ecografía abdominal ${ }^{3,4,5,11}$. Ésta, permite definir las características de la masa, su tamaño, arquitectura, localización, flujo sanguíneo y relación con estructuras adyacentes. Las lesiones sospechosas de malignidad deben ser estudiadas con $\mathrm{TC}$ o $\mathrm{RM}^{3}$. Otros métodos de imagen, como la cintigrafía con Yodo $123\left(\mathrm{I}^{123}\right)$ metaiodobenzilguanidina (MIBG), pueden ser utilizados como estudio complementario, en particular para la etapificación en $\mathrm{NB}^{11,13}$.

Existen en la literatura científica reportes y series de casos publicados sobre SPE simulando NB. Curtis y 
cols. describen, el año 1997, que por cada SPE se diagnostican 2,5 NB5. Adicionalmente, Kalenahalli y cols. reportaron un caso de SPE con presentación atípica, dado la ausencia de otras malformaciones asociadas, las que estarían presentes hasta en un $65 \%$ de los casos de $\mathrm{SPE}^{14}$. Algunos elementos orientadores de SPE en la ecografía son: localización en el espacio suprarrenal izquierdo, visualización de una arteria aberrante (generalmente desde la aorta) al Doppler, glándula suprarrenal desplazada, visible como una entidad separada, y diagnóstico durante el segundo trimestre del embarazo. En el NB lo más frecuente es observar una imagen quística compleja (50\% de los casos), sin imagen de arteria nutricia única; el diagnóstico ocurre generalmente a partir del tercer trimestre del embarazo. No es habitual visualizar la glándula suprarrenal ipsilateral ${ }^{4}$. Adicionalmente, la ausencia de metabolitos de catecolaminas en orina (ácido vanililmandélico [VMA] y ácido homovanílico [HVA]) apoyan en el diagnóstico de $\mathrm{SPE}^{3}$. El cintigrama MIBG negativo apoya el carácter benigno de la lesión, dado que más del $90 \%$ de los $\mathrm{NB}$ son ávidos por el isótopo marcado ${ }^{13}$. En el caso presentado se planteó como diagnóstico el NB dado el estudio imagenológico sospechoso y las catecolaminas urinarias en el límite de lo normal.

El buen pronóstico del NB en estadios iniciales ha llevado a cuestionarse la necesidad de resección inmediata en pacientes con masas suprarrenales detectadas prenatalmente ${ }^{6-8}$. En el control seriado de lesiones que se mantienen estables en el tiempo en cuanto a su aspecto, tamaño y marcadores en sangre $u$ orina, se ha mostrado regresión parcial o completa de las lesiones ${ }^{2}$, lo que permitiría evitar o diferir la cirugía ${ }^{6,7,15}$. Pese a que el manejo del SPE ha sido tradicionalmente quirúrgico, Yoon y cols. describen tasas significativas de regresión espontánea a 4 años, asociado a ciertos elementos imagenológicos, sugiriendo que el tratamiento quirúrgico podría considerarse en pacientes que cumplido ese plazo, no han presentado regresión o han presentado complicaciones ${ }^{9}$. En otro reporte, Obeidat et al describen un caso similar al presentado, el cual fue seguido ecográficamente hasta la resolución espontánea a los 2 años de edad ${ }^{16}$. A partir de la década del 90 comenzó a aumentar la información relativa a la posibilidad observación de pacientes seleccionados con diagnóstico de $\mathrm{NB}$, debido a que un porcentaje importante (hasta 60\%) regresan en el tiempo ${ }^{6-8}$. Los primeros protocolos de observación incluyeron a pacientes con diagnóstico de NB detectado por screening universal a los 6 meses de edad, menores de un año al momento de iniciar el seguimiento, en etapas I-II, con una lesión sólida de tamaño menor a $5 \mathrm{~cm}$ o lesión quística menor a $3.1 \mathrm{~cm}$, sin compromiso vascular y con marcadores séricos negativos ${ }^{6}$. Una serie importante sugiere que hasta $81 \%$ de los pacientes menores de 6 meses pudieran ser observados sin tratamiento quirúrgico y que el porcentaje restante tiene comportamiento benigno, con sobrevida a 3 años del $100 \% \%^{6-8}$. Otras series menores sugieren que incluso el $60 \%$ de los niños menores de un año podrían estar incluidos en esta categoría.

Nuchtern y cols. publicaron en 2012 los resultados de un protocolo prospectivo de seguimiento en pacientes menores de 6 meses con diagnóstico de NB estadio INSS I del Children's Oncology Group Study, seguidos entre 2001 y $2010^{15}$. Se controló a 83 pacientes por medio de ecografía y VMA/HVA en orina por 90 semanas, en las semanas 3-6-12-24-66 y 90 y CT/RM en 3 oportunidades. El criterio para definir necesidad de resección quirúrgica fue crecimiento mayor al 50\% del volumen inicial o aumento de metabolitos urinarios mayor al $50 \%$ del basal. Cincuenta y seis pacientes $(67,4 \%)$ completaron el periodo de observación, de los cuales el $71 \%$ tenía masa residual $<1 \mathrm{ml}$ al término del seguimiento. Dieciséis pacientes se sometieron a resección quirúrgica antes de completar el tiempo de observación, de ellos 10 tenían NB y 2 habían presentado progresión de la enfermedad. Dos pacientes tenían SPE. La sobrevida libre de eventos fue de 100 y 96\% para los pacientes con masas quísticas y sólidas respectivamente. La sobrevida libre de resección fue de $79,8 \%$ a 3 años. En $81 \%$ de los pacientes se evitó la cirugía ${ }^{15}$. En el caso que presentamos, se optó por resección precoz, considerando un grado importante de ansiedad parental y las expectativas por el establecimiento de un diagnóstico definitivo, asociado a la factibilidad técnica de realizar una cirugía mínimamente invasiva de forma expedita.

El secuestro pulmonar es una malformación congénita de la vía aérea baja. Consiste en una masa pulmonar no comunicada con el árbol tráqueo-bronquial, irrigada por una arteria derivada de la circulación sistémica $^{17}$. Las malformaciones congénitas de vía aérea pulmonar tienen una incidencia de 1: 10.000 1:35.000 recién nacidos vivos, siendo la más frecuente la malformación congénita de la vía aérea pulmonar, o CPAM por sus siglas en inglés, antes llamada malformación adenomatoídea quística o MAQ, con una incidencia de 1 en 7.200 nacidos vivos ${ }^{18}$. El secuestro pulmonar, siendo una malformación rara, representa el $0,15-6,4 \%$ del grupo ${ }^{1}$. Según su relación con el parénquima se dividen en intralobar (carecen de pleura visceral propia) y extralobar (poseen pleura visceral propia). Este último grupo da cuenta del $25 \%$ de los casos, y puede encontrarse tanto dentro como fuera del tórax (5-10\%). La mayor parte de los SPE son izquierdos y se ubican entre el lóbulo inferior del pulmón y el diafragma. Es aceptado que el $50 \%$ de ellos se asocia a otras anomalías congénitas ${ }^{11,14}$. Cada vez es más frecuente el diagnóstico antenatal por ultrasonido ${ }^{3}$. El 
SPE suele diagnosticarse temprano en la vida, mientras que el secuestro pulmonar intralobar suele tener diagnóstico más tardío, manifestándose principalmente como infecciones repetidas en un mismo sitio anatómico. Esto es infrecuente en el SPE ${ }^{17}$. En Chile existen escasas publicaciones en este tema y no hay un registro nacional de malformaciones pulmonares que permita conocer su real incidencia ${ }^{19}$. Recientemente fue publicada una serie de 17 casos de CPAM, que analiza en forma retrospectiva los casos ocurridos entre 2005 y 2016 en dos hospitales de referencia en España, y que reporta una incidencia de 1,7/10.000 nacimientos ${ }^{20}$.

La imagen típica del SPE en la ecografía es la de una masa heterogénea, usualmente piramidal. La identificación de una arteria nutricia proveniente de la aorta u otro vaso sistémico puede ayudar a establecer el diagnóstico ${ }^{11}$. A pesar de esto, establecer un diagnóstico preoperatorio es sumamente difícil y usualmente se hace con el análisis histopatológico. Si el diagnóstico es prenatal, posterior al nacimiento se debe complementar el estudio de imágenes, de preferencia con $\mathrm{TC}^{4}$.

El manejo del secuestro pulmonar tradicionalmente ha sido quirúrgico y debe ser urgente en niños que presentan dificultad respiratoria al nacer, pero puede ser diferido en niños asintomáticos o en quienes presentan infecciones recurrentes. En niños completamente asintomáticos, la decisión entre cirugía y observación es controversial, siendo la principal razón para el manejo conservador la baja tasa de complicaciones que presenta el SPE. Argumentos a favor de la cirugía precoz son la necesidad de confirmación diagnóstica (frente a otras alternativas como el NB), la disminución del riesgo de complicaciones posteriores y el mejor pronóstico cuando la cirugía se realiza antes del desarrollo de éstas ${ }^{1,2}$. La resección quirúrgica se puede hacer por videotoracoscopía o laparoscopía ${ }^{2}$. La lobectomía parcial es el tratamiento de elección en los secuestros intralobares, mientras que en SPE se realiza la resección de la masa con ligadura de los vasos ${ }^{1}$. La tasa de complicaciones es baja ${ }^{1,2}$.

El primer reporte de abordaje laparoscópico en un secuestro pulmonar data del año 2000, en un paciente de 4 meses de edad, nacido prematuramente a las 31 semanas de edad gestacional, con diagnóstico antenatal de secuestro pulmonar ${ }^{21}$. Nuevos estudios han ava- lado estos resultados, de modo que la laparoscopía es la técnica de elección ${ }^{12,22-24}$.

\section{Conclusiones}

Los tumores suprarrenales en recién nacidos son infrecuentes, y el diagnóstico diferencial incluye lesiones benignas y malignas, dentro de las cuales el más importante es el NB. El secuestro pulmonar es una malformación congénita pulmonar rara y su tratamiento de elección es quirúrgico.

A la luz de la evidencia disponible en la literatura científica, se ha puesto en duda el que todas las masas suprarrenales congénitas deban ser resecadas, especialmente aquellas asintomáticas. Sin embargo, dado la baja morbilidad de la cirugía mínimamente invasiva y la relevancia de establecer un diagnóstico, pensamos que es razonable plantear una resección precoz de este tipo de lesiones en contraposición con un manejo expectante. Los padres de los pacientes debieran conocer las alternativas de manejo, sus riesgos y beneficios, y participar en la decisión terapéutica que sea aconsejada en cada caso por el equipo tratante.

\section{Responsabilidades Éticas}

Protección de personas y animales: Los autores declaran que los procedimientos seguidos se conformaron a las normas éticas del comité de experimentación humana responsable y de acuerdo con la Asociación Médica Mundial y la Declaración de Helsinki.

Confidencialidad de los datos: Los autores declaran que han seguido los protocolos de su centro de trabajo sobre la publicación de datos de pacientes.

Derecho a la privacidad y consentimiento informado: Los autores han obtenido el consentimiento informado de los pacientes y/o sujetos referidos en el artículo. Este documento obra en poder del autor de correspondencia.

\section{Conflicto de intereses}

Los autores declaran no tener conflicto de intereses. 


\section{Referencias}

1. Andrade CF, Ferreira HPDC, Fischer GB. Congenital lung malformations. J Bras Pneumol. 2011;37(2):259-71.

2. Baird R, Puligandla PS, Laberge JM. Congenital lung malformations: informing best practice. Semin Pediatr Surg. 2011;23(5):270-7.

3. Yao W, Li K, Xiao X, Zheng S, Chen L. Neonatal suprarenal mass: differential diagnosis and treatment. J Cancer Res Clin Oncol. 2013;139(2):281-6.

4. Maki E, Oh K, Rogers S, Sohaey R. Imaging and differential diagnosis of suprarenal masses in the fetus. J Ultrasound Med. 2014;33(5):895-904.

5. Curtis MR, Mooney DP, Vaccaro TJ, et al. Prenatal ultrasound characterization of the suprarenal mass: distinction between neuroblastoma and subdiaphragmatic extralobar pulmonary sequestration. J Ultrasound Med. 1997;16(2):75-83.

6. Yoneda A, Oue T, Imura K, et al. Observation of untreated patients with neuroblastoma detected by mass screening: a "wait and see" pilot study. Med Pediatr Oncol. 2001;36(1):160-2.

7. Oue T, Inoue M, Yoneda A, et al. Profile of neuroblastoma detected by mass screening, resected after observation without treatment: results of the Wait and See pilot study. J Pediatr Surg. 2005;40(2):359-63.

8. Tanaka M, Kigasawa H, Kato K, et al. A prospective study of a long-term follow-up of an observation program for neuroblastoma detected by mass screening. Pediatr Blood Cancer. 2010;54(4):573-8.
9. Yoon HM, Kim EAR, Chung SH, et al. Extralobar pulmonary sequestration in neonates: The natural course and predictive factors associated with spontaneous regression. Eur Radiol. 2017;27(6):2489-96.

10. Martos-Moreno GA, Pozo-Román J, Argente J. Tumores suprarrenales en la infancia. An Esp Pediatr. 2013;79(3):187e16.

11. Sargar KM, Khanna G, Hulett Bowling R. Imaging of Nonmalignant Adrenal Lesions in Children. Radiographics. 2017;37(6):1648-64.

12. Rodríguez PI, Gómez-Chacón JV, Rodríguez LC, et al. Laparoscopic adrenalectomy: advantages of the minimally invasive approach. Cir Pediatr. 2016;29(4):162-5.

13. Sharp SE, Trout AT, Weiss BD, Gelfand M. MIBG in Neuroblastoma Diagnostic Imaging and Therapy. Radiographics. 2016;258-78.

14. Kalenahalli KV, Garg N, Goolahally LN, Reddy SP, Iyengar J. Infradiaphragmatic Extralobar Pulmonary Sequestration: Masquerading as Suprarenal Mass. Journal of Clinical Neonatology. 2013;2(3):146-8.

15. Nuchtern JG, London WB, Barnewolt $\mathrm{CE}$, et al. A prospective study of expectant observation as primary therapy for neuroblastoma in young infants: a Children's Oncology Group study. Ann Surg. 2012;256(4):573-80.

16. Obeidat N, Sallout B, ALAAli W. Isolated subdiaphragmatic extralobar pulmonary sequestration: masquerading as suprarenal mass with spontaneous resolution. Clin Exp Obstet Gynecol
[Internet]. 2016;43(3):457-9.

17. Bates M, Adolph V. Malformaciones congénitas pulmonares. En: Arensman R. ed. Cirugía pediátrica, Austin: Landes Bioscience. 2012;61-67.

18. Lau CT, Kan A, Shek N, Tam P, Wong $\mathrm{KKY}$. Is congenital pulmonary airway malformation really a rare disease? Result of a prospective registry with universal antenatal screening program. Pediatr Surg Int. 2017;33(1):105-8.

19. Zepeda G, Bozzo R, Aldunate M, Velozo L., Gvirtzman C. Secuestro pulmonar. Serie clínica de 16 pacientes. Rev Chil Enferm Respir. 2013;29(3):155-61.

20. Arozena MG, Rodríguez MÁ, Escudero JF, Rodríguez LM, Pérez AI, Luque JT. Diagnóstico ecográfico prenatal y resultados neonatales de la malformación adenomatoidea quística pulmonar. Rev Chil Pediatr. 2018;89(2):224-30.

21. Ferro MM, Siedman L, Feldstein VA. Minimally invasive resection of prenatally diagnosed thoracic and abdominal pulmonary sequestrations. Pediatr Endo Innov Tech. 2000; 4:137-41.

22. Danielson PD, Sherman NJ. Laparoscopic removal of an abdominal extralobar pulmonary sequestration. J Pediatr Surg. 2001;36(11):1653-5.

23. Joyeux L, Mejean N, Rousseau T, Couaillier JF, Piard F, Sapin E. Ectopic extralobar pulmonary sequestrations in children: interest of the laparoscopic approach. J Ped Surg. 2010;45(11):226973.

24. Lopes RI, Dénes FT, Bissoli J, Mendonca BB, Srougi M. Laparoscopic adrenalectomy in children. J P Urol. 2012;8(4):379-85. 